NASA-CR-204548

\title{
Applications of an energy transfer model to three problems in planetary regoliths: The solid-state greenhouse, thermal beaming, and emittance spectra
}

\author{
Bruce Hapke \\ Department of Geology and Planetary Science, University of Pittsburgh, Pittsburgh, Pennsylvania
}

\begin{abstract}
Several problems of interest in planetary infrared remote sensing are investigated using a new radiative-conductive model of energy transfer in regoliths: the solid-state greenhouse effect, thermal beaming, and reststrahlen spectra. The results of the analysis are as follows: (1) The solid-state greenhouse effect is self-limiting to a rise of a few tens of degrees in bodies of the outer solar system. (2) Non-Lambertian directional emissivity can account for only about $20 \%$ of the observed thermal beaming factor. The remainder must have another cause, presumably surface roughness effects. (3) The maximum in a reststrahlen emissivity spectrum does not occur exactly at the Christiansen wavelength where, by definition, the real part of the refractive index equals one, but rather at the first transition minimum in reflectance associated with the transition from particle scattering being dominated by volume scattering to that dominated by strong surface scattering. The transparency feature is at the second transition minimum and does not require the presence of a second band at longer wavelength for its occurance. Subsurface temperature gradients have only a small effect on emissivity bands.
\end{abstract}

\section{Introduction}

Hapke [this issue] (hereinafter refered to as paper 1) introduced a new model for thermal and radiative transfer in planetary regoliths. This model simultaneously solves the radiative transfer equation for incident visible (including the near-UV, visible, and near-IR wavelength region) and emitted thermal infrared radiation and the heat conduction equation. Approximate analytic expressions are obtained for the distribution of visible and IR radiation fluxes and the temperature as a function of depth below the surface of the medium, the visible hemispherical reflectance, and IR directional and hemispherical emissivities of a medium of anisotropically scattering particles. The solid-state greenhouse effect and the contribution of radiation to the thermal conductivity appear intrinsically in the model with no ad hoc additions.

In this paper the new model is applied to three problems of interest in the remote sensing of planetary regoliths. Only time-independent solutions are considered. Section 2 analyzes the solid-state greenhouse in outer planet satellites. Section 3 treats the effect of a non-Lambertian emissivity on thermal beaming. Section 4 discusses the relation between the spectra of the refractive index and emissivity in a reststrahlen band.

The notation used in this paper is identical to that in paper 1, and all the equations are taken exactly from that reference, which should be consulted for notation and definitions.

\section{The Solid-State Greenhouse Effect}

An atmospheric greenhouse effect can occur on a planet whose atmosphere is transparent to visible light but which absorbs thermal IR radiation. The energy of visible sunlight is absorbed at the ground level but is radiated back into space as

Copyright 1996 by the American Geophysical Union.

Paper number 96JE00918.

0148-0227/96/96JE-00918\$09.00
IR radiation from upper layers of the atmosphere. This requires a temperature gradient to transport heat from the surface to the radiating layer. On Venus this effect produces a surface temperature that is nearly triple the radiative equilibrium temperature. Brown and Matson [1987] and Matson and Brown [1989] have pointed out that a strong solid-state greenhouse effect could occur in a planetary regolith that is weakly absorbing in the visible and strongly absorbing in the IR and that this would have important implications for the interior temperatures of icy satellites. However, they described the visible radiation source term by approximate, empirical expressions, rather than using the radiative transfer equation, so that their results are quantitatively uncertain.

The results of the radiative equilibrium calculation of paper 1 will be used to calculate the subsurface temperature rise in a regolith in equilibrium with sunlight. Equation (58) of paper 1 for the reduced temperature (temperature relative to the blackbody radiative equilibrium temperature $T_{r}$ ) at infinite optical depth below the surface is

$$
\begin{aligned}
T^{*}(\infty)= & T_{s}^{*}-\pi q T^{* 4}(\infty) \\
& +q\left[\pi \varphi_{T s}^{*}+\frac{1}{R}\left(\gamma_{v}^{* 2} \mu_{0 v}^{* 2} A+\frac{B}{4}\right)\right],
\end{aligned}
$$

where all quantities are defined in paper $1 ; \varphi_{T s}^{*}, A$, and $B$ are given by equations $(56),(50 \mathrm{~b})$, and $(50 \mathrm{c})$ of paper 1 , respectively, $T_{s}^{*}$ is the reduced surface temperature, given by (61) and (56) of paper $1 ; q=J_{v} / E_{T}^{*} k T_{r}=\sigma_{0} T_{r}^{3} / \pi E_{T}^{*} k, J_{v}$ is incident visible irradiance (including the near-UV, visible, and nearIR), $\sigma_{0}$ is Boltzmann's constant, $T_{r}$ is blackbody radiative equilibrium temperature, $E_{T}^{*}$ is reduced extinction coefficient of the regolith, and $k$ is solid-state thermal conductivity.

It is instructive to first calculate the temperature for the relatively simple case where the particles of the regolith are large compared to the wavelength and scatter isotropically. Then several of the quantities that occur in the parameters in 
(1) can be simplified by putting $Q_{E v}=Q_{E T}=1, \beta_{v}=\beta_{T}=$ $0, E_{v}=E_{r}$, and $R=\zeta_{v}=\zeta_{T}=1$. After substituting the appropriate expressions from paper 1 for $A, B$, and $\varphi_{T s}^{*}$, the quantities inside the square brackets in (1) can be factored and combined to yield

$$
T^{*}(\infty)=T_{s}^{*}-\pi q T^{* 4}(\infty)+q \mu_{0}\left(1+2 \mu_{0}\right) / 2,
$$

where $\mu_{0}=\cos i$ and $i$ is angle of incidence.

In both (1) and (2) the first term on the right-hand side is the reduced surface temperature, while the second term accounts for heat transfer by thermal radiation. The third term describes the relative rise in subsurface temperature, that is, the greenhouse effect. Note that for the simplified conditions of (2) this term is independent of both the visible and thermal constants of the medium and is directly proportional to the incident irradiance $J_{v}$. It has its maximum value of $3 q / 2$ when $\mu_{0}=1$. At the distance of Jupiter, a regolith having a high single scattering albedo, but otherwise like lunar soil, would have $q$ 0.023 (see paper 1 ), so that $3 q / 2 \sim 0.035$, corresponding to a greenhouse effect of only a few percent. The reason the relative temperature rise is so small is that although a small absorption coefficient allows the visible radiant power to be deposited deep in the regolith, it also causes a high visual albedo, so that less power enters the medium. The two effects exactly cancel each other when the particles are isotropic scatterers.

Hence small absorption in the visible combined with high IR opacity is not, by itself, sufficient to cause a large solid-state greenhouse effect in a regolith. However, the temperature rise can be increased if the particles are forward scattering, since then both the absorbance and the albedo can be small in the visible. To calculate the rise in temperature in this case, the full equation (1) from (58) of paper 1 must be used. Substituting from paper 1 , factoring, collecting terms, and reverting to unstarred (unreduced) quantities gives

$$
\begin{aligned}
& T(\infty)=T_{s}-\frac{\sigma_{0}}{\zeta_{T}^{2} E_{T} k} T^{4}(\infty)+\frac{J_{v}}{2 \zeta_{T}^{2} E_{T} k} \frac{\zeta_{v}^{2} \mu_{0}}{\left(1+2 \zeta_{v} \gamma_{v} \mu_{0}\right)\left(1-\beta_{v}\right)} \\
& \cdot\left\{\left[\left(\frac{\gamma_{v}}{\zeta_{v}}+2 \zeta_{v} \gamma_{v} \mu_{0}\right)+\beta_{v} \frac{\gamma_{v}}{\zeta_{v}}\left(1-2 \frac{\gamma_{v}}{\zeta_{v}}-2 \gamma_{v}^{2} \mu_{0}\right)\right]\right. \\
& +\frac{E_{T}}{E_{v}} \frac{\zeta_{T}^{2}}{\zeta_{v}^{2}}\left[\left(1+2 \zeta_{v}^{2} \mu_{0}\right)\left(1-\frac{\gamma_{v}}{\zeta_{v}}+2 \zeta_{v} \gamma_{v} \mu_{0}\right)\right. \\
& \left.\left.+\beta_{v}\left(1-2 \zeta_{v} \gamma_{v} \mu_{0}-\frac{\gamma_{v}}{\zeta_{v}}-2 \gamma_{v}^{2} \mu_{0}-2 \frac{\gamma_{v}^{3} \mu_{0}}{\zeta_{v}}-4 \zeta_{v} \gamma_{v}^{3} \mu_{0}^{2}\right)\right]\right\},
\end{aligned}
$$

where all quantities are defined in paper 1.

For realistic optical properties of a strongly forward scattering, high-albedo material in the visible, the data for dry Antarctic snow measured by Schwerdtfeger and Weller [1977] will be used. They found that between the surface and a depth of 100 $\mathrm{cm}$ the mean size of the snow particles increased from about $0.2 \mathrm{~mm}$ at the surface to $1 \mathrm{~mm}$ at $50 \mathrm{~cm}$. The intensity of visible light decreased according to the relation $I=I_{0} \exp (-\chi z)$, where $z$ is the depth below the surface of the snow and $\chi d=$ 0.03 , where $d$ is the grain size. The bulk density was $\rho=0.30$. The visual albedo was 0.83 , and the power of incident sunlight per unit area on the snow was $900 \mathrm{cal} \mathrm{cm} \mathrm{cm}^{-2} \mathrm{~d}^{-1}$. Since the irradiance of sunlight is $2800 \mathrm{cal} \mathrm{cm} \mathrm{cm}^{-2} \mathrm{~d}^{-1}$, the cosine of the angle of incidence at the time of the measurements was $\mu_{0}=$ $900 / 2800=0.32$.

The relative flux of visible light inside the snowpack is described by equation (49) of paper 1 for $\varphi_{v}^{*}$. This equation consists of two terms. The first term describes the incident unscattered sunlight and is negligible deeper than a few particle diameters. Below this depth only the second term remains, which describes the multiply scattered sunlight and is proportional to $\exp \left(-2 \gamma^{*} \tau^{*} \tau^{*}\right)$. Hence $\chi^{z}$ can be identified with $2 \gamma_{v}^{*} \tau_{v}^{*}=2 \zeta_{v} \gamma_{v} E_{v} z$, so that $\chi=2 \zeta_{v} \gamma_{v} E_{v}$.

Now, $E_{\eta}=N \sigma Q_{E v}$, where $N$ is number of particles per unit volume, $\sigma$ is particle cross sectional area, and $Q_{E v}$ is extinction efficiency. Since the particles are large compared with both visible and thermal radiation, $Q_{E_{3}} \simeq Q_{E T} \simeq 1$. The bulk density is $\rho=\rho_{s} N \pi d^{3} / 6$, so $N=6 \phi / \pi d^{3}$, where $\phi=\rho / \rho_{s}$ is the filling factor; since $\rho_{s} \simeq 1, \phi \simeq 0.30$. Thus $E_{v}=$ $N \sigma Q_{E v} \simeq\left(6 \phi / \pi d^{3}\right) *\left(\pi d^{2} / 4\right) * 1=3 \phi / 2 d$, so that $\chi d=$ $2 \zeta_{\nu} \gamma_{v}(3 \phi / 2 d) d=0.03$, and $\zeta_{v} \gamma_{\nu}=0.033$. This gives one relation between $\beta_{v}$, and $w_{v}$. A second relation involving $\beta_{v}$ and $w_{y}$ is the measured albedo of the snow, $A_{h}=0.83$ at $\mu_{0}=0.32$, which is given by equation (65) of paper 1 . The two expressions can be solved simultaneously to give $w_{y}=0.997$ and $\beta_{v}=0.672$, so that $\zeta_{v}=0.574$ and $\gamma_{v}=0.0574$.

The IR optical properties of snow are poorly known. Irvine and Pollack [1968] estimate on the basis of highly uncertain data that in the 10-25 $\mu \mathrm{m}$ wavelength range a perfect sphere of water ice with radius $10 \mu \mathrm{m}$ has $w \sim 0.8$ and has an average cosine of the scattering angle -0.7 . However, Salisbury et al. [1994a] measured the IR reflectance of water frost to be less than $1 \%$, which implies that $w$ must be very small there. Thus $\gamma_{T}$ cannot be greatly different from 1 . Particles are strongly forward scattering only if they are perfect, isolated, clear spheres [McGuire and Hapke, 1995]. Rough-surfaced, irregular, absorbing particles have scattering patterns that are much less asymmetric. Hence $\zeta_{T}$ is probably also close to 1 .

These values will be used to calculate the solid-state greenhouse effect expected for a similar material on the surface of an icy satellite of Jupiter. Substituting them into equation (3) and taking $\mu_{0}=1$ to maximize the greenhouse effect, the coefficient of $J_{v} / \zeta_{T}^{2} E_{T} k$ is found to have the value 3.19. Following Brown and Matson [1987], it will be assumed that $k=$ $0.001 \mathrm{~W} \mathrm{~m}^{-1} \mathrm{~K}^{-1}$. At $D=5 \mathrm{AU}, J_{v}=1360 / 25=54.4 \mathrm{~W} \mathrm{~m}^{-2}$, so that $T_{r}=\left(\pi J_{v} / \sigma_{0}\right)^{1 / 4}=234 \mathrm{~K}$. From equation (65) of paper $1, \varphi_{T s}^{*}=\mu_{0}\left(1-A_{h}\right) / 2 \pi=0.0271$, and $T_{s}=$ $T_{r}\left[\left(1+\gamma_{T}^{*}\right) \varphi_{r_{s}}^{*} / \gamma_{T}^{*}\right]^{1 / 4}=113 \mathrm{~K}$. Also, $E_{T}=E_{\vartheta}=3 \phi / 2 d$; it will be assumed that $\phi=0.30$, but $d$ will be allowed to vary, so that $E_{v}=0.450 / d \mathrm{~m}^{-1}$.

When these values are inserted into (3), the temperature deep within the snow pack is found to be given by

$T(\infty)=T_{s}+3.86 \times 10^{5} d-1.26 \times 10^{-4} d T^{4}(\infty) \mathrm{K}$,

where $T_{s}=113 \mathrm{~K}$ is the surface temperature and $d$ is the particle size in meters. This expression may be readily solved for $T(\infty)$. The result is plotted as the solid line in Figure 1, which shows the rise in temperature $\Delta T=T(\infty)-T_{s}$ versus regolith particle size for $10 \mu \mathrm{m} \leq d \leq 1 \mathrm{~cm}$. For $d$ of the order of a few tens of micrometers, similar to lunar regolith, $\Delta T \sim$ $10 \mathrm{~K}$. For $d \sim 1 \mathrm{~mm}$, similar to the Antartic dry snow, $\Delta T \sim$ $100 \mathrm{~K}$. For $d \sim 1 \mathrm{~cm}$, such as in a wet, terrestrial snowfall, $\Delta T \sim 120 \mathrm{~K}$. The temperature rise saturates at $\Delta T \sim 120 \mathrm{~K}$ for larger $d$. As $d$ increases, the temperature increases, but the radiative conductivity also increases and limits the rise.

These are the greenhouse temperature rises on a nonrotat- 
ing body. However, real bodies rotate with respect to the Sun, so that the greenhouse rise in the deep interior is proportional to the value of the time-dependent visible irradiance averaged over time. To a first approximation, this radiance is proportional to $\cos i$ averaged over the period of rotation, $\langle\cos i\rangle \sim$ $1 / \pi$. Hence the temperature rise on a rotating body will be reduced by roughly a factor of $1 / \pi$, which is indicated by the dashed line in Figure 1. This temperature increase does not exceed $40 \mathrm{~K}$.

Moreover, the low thermal inertias and inferred low thermal conductivities [Brown and Matson, 1987] imply that the regolith particle sizes on the icy satellites are probably small, similar to lunar soil, so that the solid-state greenhouse effects that might be expected there are relatively modest, a few tens of degrees.

Temperature rises of the order of $40 \mathrm{~K}$ are certainly not negligible and, as Matson and Brown [1989] emphasize, could have important implications for the interior structures of the bodies. However, this analysis does not confirm the potential increases of $400 \mathrm{~K}$ and more on icy Jovian satellites estimated by their models. Matson and Brown, of course, recognized that such high temperatures were physically impossible in icy regoliths but argued that sublimation, melting, and the resulting mass transport were the processes that kept the temperatures low. Fanale et al. [1990, p. 203] asserted that "If (and only if) the effects of latent heat, mass transport and densification are taken into account, we can reconcile the magnitude of the theoretically predicted greenhouse effect with the observed morphology of Europa ...".

However, a solid-state greenhouse effect is intrinsically different from an atmospheric greenhouse effect, because in the latter the optical depth at which visible energy is deposited is essentially independent of the bolometric albedo, whereas in the former, the two are tightly coupled. The Matson and Brown [1989] model did not adequately take this coupling into account, nor did it appreciate that radiation can contribute to the thermal conductivity even at temperatures below $200 \mathrm{~K}$. Melting and sublimation may very well be occurring in the interior of Europa, as they hypothesize, but these processes are not necessary to limit the greenhouse temperature rise.

\section{Thermal Beaming}

The radiometric technique [Morrison and Lebofsky, 1979] is a major method for determining the diameters and physical albedos of objects that are too small to be resolved. It is especially important in the study of asteroids. This method involves comparing measured radiances in the visible and thermal IR received from the object at small phase angles. Early models, which assumed that surfaces radiated in the IR in accordance with Lambert's law, yielded diameters that were systematically too large and albedos that were too small. It is known that many surfaces, notably the Moon's, are not Lambertian emitters; hence an empirical parameter to correct for this, called the thermal beaming factor, was introduced. Most explanations of the nature of the beaming factor, particularly that of Spencer [1990], have concentrated on the effects of unresolved surface roughness. However, neither the visible reflectance nor the thermal emission from the surface of a particulate medium are Lambertian, so that it is of interest to enquire as to the importance of their contributions to thermal beaming.

To estimate the magnitude of these effects it will be sufficient to consider only the so-called standard model, in which it is

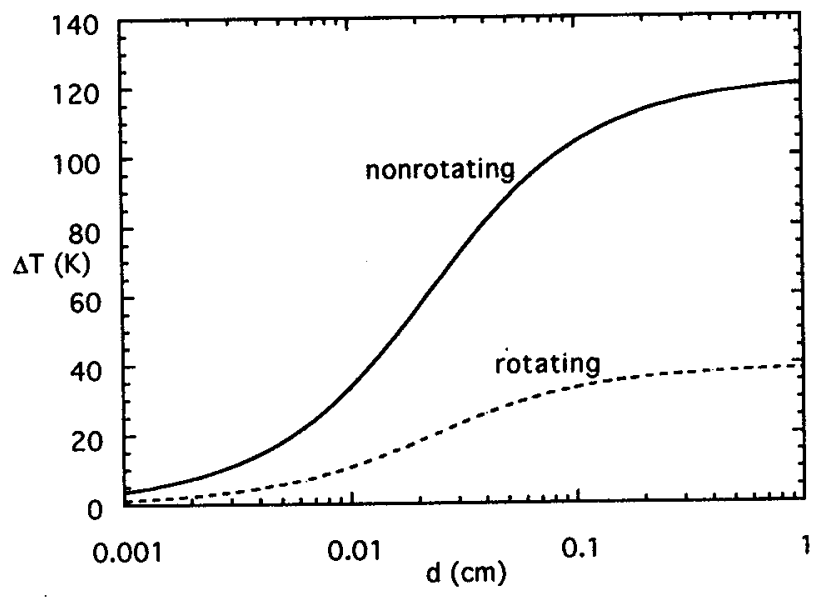

Figure 1. Increase in temperature due to the solid-state greenhouse effect, calculated from equation (4), plotted against regolith particle size. The solid line shows $\Delta T$ on a nonrotating body, and the dashed line shows $\Delta T$ on a rotating body.

assumed that the body does not rotate and the regolith is in radiative equilibrium. The visible power scattered by a spherical body into zero phase angle is, by definition,

$$
P_{v}=J_{v} \pi \mathscr{R}^{2} \frac{1}{\pi} A_{p v}=J_{v} \mathscr{R}^{2} A_{p v}=J_{v} \mathscr{R}^{2} A_{s v} / \xi_{v}
$$

where $\mathscr{R}$ is the radius of the body, $A_{p y}$ is the visual physical albedo, $A_{s v}$ is the visual spherical (Bond) albedo, and $\xi_{v}=$ $A_{p v} / A_{s v}$ is the visual phase integral.

The thermal power scattered by the body into zero phase angle is

$$
\begin{aligned}
P_{T} & =\int_{\mathscr{A}} I_{T}(i=e, e) \mu d \mathscr{A} \\
& =\int_{0}^{\pi / 2} \varepsilon_{d}(\mu) \frac{\sigma_{0}}{\pi} T_{e}(\mu) \mu 2 \pi \mathscr{R}^{2} \sin e d e
\end{aligned}
$$

where $A$ is the illuminated area on the surface of the body and $T_{c}$ is the effective temperature at which the surface radiates to space. On any illuminated unit area of the surface in equilibrium,

$$
\varepsilon_{h} \frac{\sigma_{0}}{\pi} T_{e}^{4}\left(\mu_{0}\right)=J_{v} \mu_{0}\left[1-A_{h v}\left(\mu_{0}\right)\right]
$$

so that at zero phase where $\mu_{0}=\mu$,

$$
P_{T}=2 \pi \mathscr{R}^{2} J_{v} \int_{0}^{1} \frac{\varepsilon_{d}(\mu)}{\varepsilon_{h}}\left[1-A_{h v}(\mu)\right] \mu^{2} d \mu
$$

Combining (5) and (7) gives

$$
\frac{P_{T}}{P_{y}}=2 \pi \frac{\xi_{v}}{A_{s v}} \int_{0}^{1} \frac{\varepsilon_{d}(\mu)}{\varepsilon_{h}}\left[1-A_{h v}(\mu)\right] \mu^{2} d \mu .
$$

If the surface were perfectly Lambertian, the following would be true: (1) $\varepsilon_{d}(\mu)$ is independent of $e$ and equal to $\varepsilon_{h}$; (2) $A_{h y}\left(\mu_{0}\right)$ is independent of $i$ and equal to $A_{s v} ;$ (3) the 


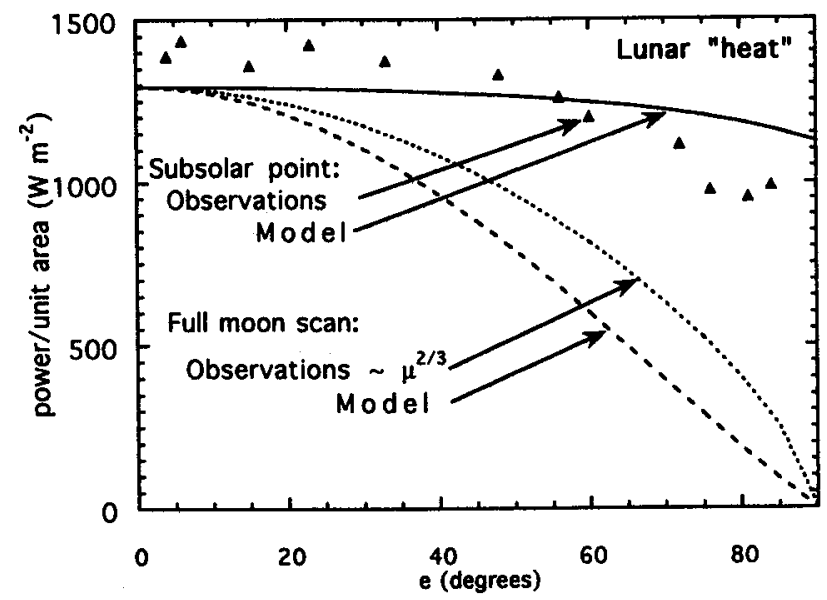

Figure 2. Measured effective power per unit area emitted by the lunar surface as a function of the angle of emergence $e$ compared with theoretical models of a regolith with a macroscopically smooth surface. The top points and curve correspond to emission from the subsolar point at different $e$. The bottom two curves correspond to a scan across the full Moon.

surface radiates according to Lambert's law, so that $T_{c}^{4}\left(\mu_{0}\right)=$ $T_{e}^{4}(i=0) \mu_{0}$. Then the integral in (7) and (8) can be evaluated to give

$$
P_{T}=2 \pi \mathscr{R}^{2} J_{v}\left(1-A_{s \nu}\right) / 3
$$

and

$$
P_{t} / P_{v}=(2 \pi / 3) \xi_{v}\left(1-A_{s v}\right) / A_{s v}
$$

To correct for non-Lambertian effects, equation (6) is modified by multiplying the left-hand side by an empirical thermal beaming parameter $\eta$, while keeping $\varepsilon_{d}(\mu)=\varepsilon_{h}$ and $A_{h v}\left(\mu_{0}\right)$ $=A_{s v} ;$ thus

$$
\eta \varepsilon_{h} \frac{\sigma_{0}}{\pi} T_{c}^{4}\left(\mu_{0}\right)=J_{\nu} \mu_{0}\left(1-A_{s v}\right)
$$

Then (7) and (8) become

$$
P_{T}=2 \pi \mathscr{R}^{2} J_{v}\left(1-A_{s v}\right) / 3 \eta
$$

and

$$
P_{T} / P_{v}=(2 \pi / 3) \xi_{v}\left(1-A_{s v}\right) / \eta A_{s v}
$$

A value is assumed for $\xi_{v}$ (often the lunar value), and, assuming $\eta$ is known, equation (11) can be solved for $A_{s v}$ and $A_{p v}=$ $\xi_{v} A_{s v} ;$ then $\mathscr{R}$ can be found from (5).

Requiring that (7) and (10) for $P_{T}$ be equal gives the following expression for $\eta$ :

$$
\eta=\frac{1}{3} \frac{1-A_{s v}}{\int_{0}^{1} \frac{\varepsilon_{d}(\mu)}{\varepsilon_{h}}\left[1-A_{h v}(\mu)\right] \mu^{2} d \mu}
$$

The value of $\eta$ based on observations of the Moon and the Galilean satellites is $\eta=0.75$ [Lebofsky et al., 1986].

In order to evaluate the contribution of the intrinsic nonLambertian behavior of $\varepsilon_{d}(\mu)$ and $A_{h v}\left(\mu_{0}\right)$ of a particulate medium, the Moon will be taken to be a representative body. Equation (69) of paper 1 was used to numerically calculate the radiance $I_{T}(i=0, e)$ emitted by the subsolar point as it moves across the Moon's surface during a lunation. The same equation was used to calculate the distribution of radiance $I(i=e$, $e$ ) across the full Moon. The equations derived in section 4 of paper 1 were used to calculate the various quantities in (12) and $\eta$ was evaluated. The bolometric albedo of the Moon was taken to be $A_{s v}=0.12$ [Lane and Irvine, 1973], and the hemispherical emissivity $\varepsilon_{h}=0.89$ [Saari et al., 1972]. A value was assumed for $\boldsymbol{\beta}_{y}$, and $w_{y}$ was found from $A_{s v}$, following which $A_{h}\left(\mu_{0}\right)$ was calculated. Similarly, a value was assumed for $\beta_{T}, w_{T}$ was found from $\varepsilon_{h}$, and then $\varepsilon_{d}(\mu)$ was calculated. $I_{T}(i, e) /$ was then found from $A_{h}\left(\mu_{0}\right)$ and $\varepsilon_{d}(\mu)$.

The calculated predictions were compared with the lunar thermal data reported by Sinton [1961] and Saari and Shorthill [1972]. The results are shown in Figure 2. The triangles are Sinton's measurements of "planetary heat," which is the power per unit area emitted by the subsolar point as it moves across the lunar surface during a lunation inferred from thermal IR measurements, assuming that the Moon radiates in accordance with Lambert's law. However, if the Moon radiated like a perfect Lambert surface, the emitted power per unit area would be independent of $e$. The observed limb darkening is an indication of departures from Lambertian behavior. The model curve exhibits some limb darkening, but not nearly as much as the Moon.

The observed [Saari and Shorthill, 1972] power distribution across the full moon is approximately proportional to $\mu^{2 / 3}$, whereas for a Lambert surface the power would be proportional to $\mu^{1}$. Figure 2 shows the power per unit area calculated from the model and according to $\mu^{2 / 3}$. The amount of limb darkening predicted by the model is too large.

The thermal beaming factor predicted by the model was calculated by numerical integration of (12) and found to be $\eta=0.95$.

The values of the asymmetry parameters used in calculating the curves of Figure 2 are $\beta_{v}=\beta_{T}=0$. Other values were tried, but the resulting curves were found to be quite insensitive to this parameter. The value of $A_{s v}$ was varied between 0.09 and 0.15 and of $\varepsilon_{h}$ from 0.85 to 0.95 , again with only minor effects.

Hence although the angular emitting and scattering properties of particulate media are not Lambertian, the departures are insufficient to explain the observations. In particular, they account for only $20 \%$ of the departure of $\eta$ from unity. The Moon exhibits a surge in visible brightness at full Moon, and it may be speculated that a similar effect might occur in the IR. However, the visible opposition effects from planetary regoliths result from either or both coherent (coherent backscatter) or incoherent (shadow hiding) phenomenon in which the direction of the incident radiation plays a special role. Since there is no external source of thermal radiation, analogous phenomena cannot occur in the IR. Thus the other $80 \%$ of $\eta$ is most likely due to macroscopic roughness [Spencer, 1990].

\section{Spectral Emissivity}

The location and contrast in features of emissivity spectra are important for infrared remote sensing and have been the subject of considerable recent attention. Conel [1969] and Logan and Hunt [1970] identified maxima in the emissivity reststrahlen spectra of particulate materials with the Christiansen wavelength and pointed out that this wavelength was diagnostic of composition. Since then these maxima have been investigated in detail by several others, including Salisbury [1993], 
Salisbury and Walter [1989], B. Henderson and B. Jakosky Near-surface thermal gradients and mid-IR spectra: A new model including scattering and application to real data, (submitted to Journal of Geophysical Research, 1995), and Wald and Salisbury [1995]. A particular concern has been the effect of subsurface temperature gradients on the wavelength of the maxima. Henderson and Jakosky [1994, also submitted manuscript, 1995] concluded that such temperature gradients had only minor effects.

The formalism developed in previous sections of this paper will be used to investigate this question further. Other authors used the spectra of real minerals. However, such spectra usually consist of multiple overlapping bands, which tends to obscure important properties of the spectra. Hence the Lorentz model of absorption bands will be used to generate theoretical emissivity spectra of media in radiative equilibrium with sunlight in order to clarify and elucidate the nature of the features of a strong reststrahlen band seen in emissivity. Lorentz theory [e.g., Wooten, 1972] is widely accepted as providing a reliable description of such bands [e.g., Spitzer and Kleinman, 1961].

According to the Lorentz model, the complex dielectric constant (also called the dielectric function) in the vicinity of a strong absorption band is given by

$$
K_{e}=K_{r}+i K_{i}
$$

where

$$
\begin{gathered}
K_{r}=n_{c}^{2}+\frac{\nu_{p}^{2}\left(\nu_{0}^{2}-\nu^{2}\right)}{\left(\nu_{0}^{2}-\nu^{2}\right)^{2}+\Xi^{2} \nu^{2}}, \\
K_{i}=\frac{\nu_{p}^{2} \Xi \nu}{\left(\nu_{0}^{2}-\nu^{2}\right)^{2}+\Xi^{2} \nu^{2}},
\end{gathered}
$$

$i=\sqrt{-1}, n_{c}$ is the continuum real part of the refractive index outside of the band and $v$ is the frequency; the band is characterized by three parameters: the frequency of the band center $\nu_{0}$, the plasma frequency $\nu_{p}$, and the collision frequency $\Xi$.

By definition, the dielectric constant is the square of the complex refractive index,

$$
m=n_{r}+i n_{i}
$$

so that

$$
K_{e}=K_{r}+i K_{i}=n_{r}^{2}-n_{i}^{2}+i 2 n_{r} n_{i}
$$

which may be solved for $n_{r}$ and $n_{i}$ to give

$$
\begin{aligned}
& n_{r}=\left\{\frac{1}{2}\left[\left(K_{r}^{2}+K_{i}^{2}\right)^{1 / 2}+K_{r}\right]\right\}^{1 / 2} \\
& n_{i}=\left\{\frac{1}{2}\left[\left(K_{r}^{2}+K_{i}^{2}\right)^{1 / 2}-K_{r}\right]\right\}^{1 / 2} .
\end{aligned}
$$

In order to calculate the emissivity, a model for the wavelength-dependent single scattering albedo $w_{\lambda}$ and angular scattering function $p_{\lambda}(g)$ of the medium is needed. It will be assumed that the particles are all equant and of a single size $d$ large compared with the wavelength $\lambda$. In that case, $Q_{E \lambda}=1$ and the single scattering albedo $w_{\lambda}$ is equal to the particle scattering efficiency $Q_{s \lambda}$. Mie theory will give incorrect results if it is used to calculate $w_{\lambda}$ because it applies only to an isolated sphere and includes diffraction, which is inappropriate for particles in a close packed medium [Hapke, 1993]. Since the particles are large compared to the wavelength, geometrical optics, or ray theory, is appropriate. Hapke [1993] showed that the following expression is a good approximation for $w_{\lambda}$ when ray theory is applicable,

$$
w(\lambda)=S_{e}+\frac{\left(1-S_{e}\right)^{2}}{1-S_{e} e^{-\alpha d}} e^{-\alpha d}
$$

where

$$
\alpha=4 \pi n_{i} / \lambda
$$

is the absorption coefficient and $S_{e}$ is the coefficient for reflection of radiation externally incident on the surface from all directions,

$$
S_{e}=\int_{0}^{\pi}\left[R_{1}(\vartheta)+R_{2}(\vartheta)\right] \cos \vartheta \sin \vartheta d \vartheta
$$

where $R_{1}(\vartheta)$ and $R_{2}(\vartheta)$ are the Fresnel reflection coefficients [Born and Wolf, 1980],

$$
\begin{gathered}
R_{1}(\vartheta)=\frac{\left[\cos \vartheta-G_{1}\right]^{2}+G_{2}^{2}}{\left[\cos \vartheta+G_{1}\right]^{2}+G_{2}^{2}}, \\
R_{2}(\vartheta)=\frac{\left[\left(n_{r}^{2}-n_{i}^{2}\right) \cos \vartheta-G_{1}\right]^{2}+\left[2 n_{i} n_{i} \cos \vartheta-G_{2}\right]^{2}}{\left[\left(n_{r}^{2}-n_{i}^{2}\right) \cos \vartheta+G_{1}\right]^{2}+\left[2 n, n_{i} \cos \vartheta+G_{2}\right]^{2}},
\end{gathered}
$$

$$
\begin{aligned}
G_{1}(\vartheta)= & \left\{\frac { 1 } { 2 } \left[\left(\left(n_{r}^{2}-n_{i}^{2}-\sin ^{2} \vartheta\right)^{2}+4 n_{r}^{2} n_{i}^{2}\right)^{1 / 2}\right.\right. \\
& \left.\left.+\left(n_{r}^{2}-n_{i}^{2}-\sin ^{2} \vartheta\right)\right]\right\}^{1 / 2}, \\
G_{2}(\vartheta)= & \left\{\frac { 1 } { 2 } \left[\left(\left(n_{r}^{2}-n_{i}^{2}-\sin ^{2} \vartheta\right)^{2}+4 n_{r}^{2} n_{i}^{2}\right)^{1 / 2}\right.\right. \\
& \left.\left.-\left(n_{r}^{2}-n_{i}^{2}-\sin ^{2} \vartheta\right)\right]\right\}^{1 / 2} .
\end{aligned}
$$

In $(15)$ the contributions of different phenomena to particle scattering are explicitly separated. The first term of (15) describes radiation that has been reflected by the surface of the particle, while the second term describes light that has been refracted into the particle and partially absorbed there or scattered back out from within its volume. All orders of internal scattering are included.

Although transparent spherical particles are strongly forward scattering, highly absorbing ones are not. Furthermore, McGuire and Hapke [1995] have shown that any departures from a perfect sphere, such as irregular shape or surface roughness causes the particle phase function to be more isotropic. Hence, for simplicity, it will be assumed that the particles scatter isotropically in both the visible and IR, so that the particle phase function $p_{\lambda}(g)=1$.

Since the particles are large compared to the wavelength, the extinction coefficient $E_{\lambda}$ will not vary appreciably in the thermal IR so that $\tau_{\lambda} \simeq \tau_{T}$. Then for isotropic particles the thermal radiance emerging from the surface of the medium at wavelength $\lambda$ and angle $e$ is [Hapke, 1993]

$$
I_{\lambda}(\mu)=\int_{0}^{\infty}\left[w_{\lambda} \varphi_{\lambda}\left(\tau_{T}\right)+\frac{\gamma_{\lambda}^{2}}{\pi} U_{\lambda}(T)\right] e^{-\tau / \mu} d \tau_{T} / \mu,
$$

where $\varphi_{\lambda}\left(\tau_{T}\right)$ is the average isotropic spectral radiance per unit wavelength interval,

$$
\begin{gathered}
\gamma_{\lambda}=\sqrt{1-\overline{w_{\lambda}}} \\
U_{\lambda}(T)=\left(2 \pi h_{0} c_{0} / \lambda^{5}\right)\left(e^{h_{\left(\mid c_{0} / k\right) \lambda}-1}-1\right)^{-1}
\end{gathered}
$$



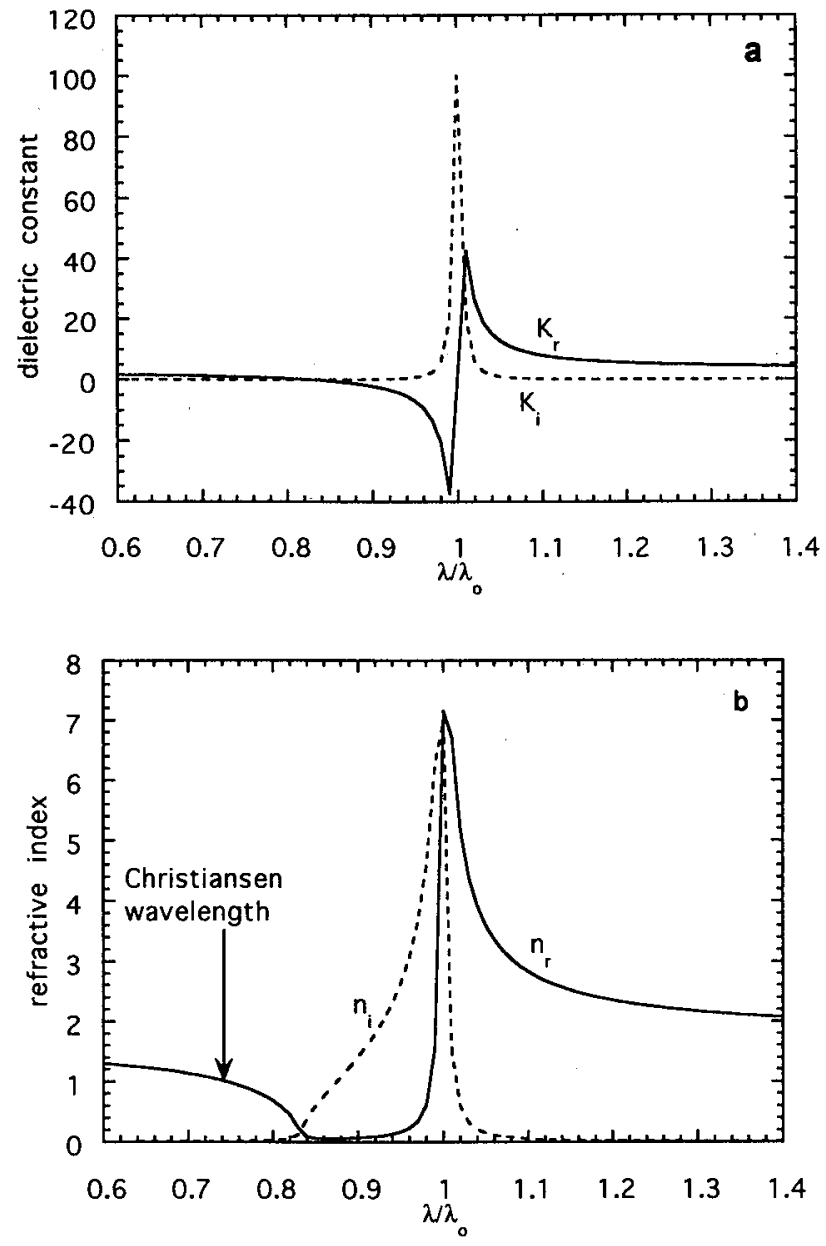

Figure 3. Lorentz model spectrum of a reststrahlen band with parameters $\nu_{p} / \nu_{0}=1.00, \Xi / \nu_{0}=0.01, n_{c}=1.50$. The band center is at $\lambda_{0}$ : (a) dielectric constant and (b) refractive index.

is the Planck function, $h_{0}$ is Planck's constant, $c_{0}$ is the speed of light in vacuum, and $k_{0}$ is Boltzmann's constant.

The wavelength-dependent boundary layer approximation for the radiance is given by equation (75) of paper 1 , which for isotropic scatterers $\left(\zeta_{\lambda}=1\right)$ is

$$
\varphi_{\lambda}\left(\tau_{T}\right)=\frac{1}{\pi} U_{\lambda}(T)-\frac{\varphi_{\lambda s}}{\gamma_{\lambda}} e^{-2 \gamma_{T T T}}
$$

where

$$
\begin{gathered}
\varphi_{\lambda s} \simeq \frac{\gamma_{\lambda}}{1+\gamma_{\lambda}} \frac{1}{\pi} U_{\lambda}\left(T_{s}\right), \\
T\left(\tau_{T}\right)=T_{r} T^{*}\left(\tau_{T}^{*}\right),
\end{gathered}
$$

$T^{*}\left(\tau_{r}^{*}\right)$ is given by equation (60) of paper 1 , which describes the temperature regime in a medium in radiative equilibrium with sunlight, and

$$
T_{s}=T_{r} T_{s}^{*}
$$

where $T_{s}^{*}$ is given by equations (61) and (56) of paper 1 .

This system of equations was converted to wavelength as the independent variable and evaluated numerically with the following parameters: $n_{c}=1.50, \nu_{p} / \nu_{0}=1.00, \Xi / \nu_{0}=0.01$, $\lambda_{0}=c_{0} / \nu_{0}=10 \mu \mathrm{m}, d / \lambda_{0}=10, w_{v}=0.36, \beta_{v}=\beta_{T}=$ 0 . The spectra of the resulting dielectric constant and refractive index are shown in Figures $3 \mathrm{a}$ and $3 \mathrm{~b}$, respectively, and the single scattering albedo is shown in Figure 4.

Four cases of interest were studied: $q=0.26, \mu_{0}=1$, $T_{r}=524 \mathrm{~K}, w_{T}=0.52$, corresponding to the subsolar point on the Moon viewed normally; $q=0.26, \mu_{0}=\cos 60^{\circ}, T_{r}=$ $524 \mathrm{~K}, w_{T}=0.52$, corresponding to a point on the lunar surface $60^{\circ}$ away from the subsolar point and viewed normally; $q=1.067, \mu_{0}=1, T_{r}=839 \mathrm{~K}, w_{T}=0.52$, corresponding to the subsolar point on Mercury viewed normally; and $q=$ $0.023, \mu_{0}=1, T_{r}=234 \mathrm{~K}, w_{T}=0.49$, corresponding to the subsolar point on a Jovian satellite viewed normally. These parameters were chosen in order to vary the subsurface temperature gradient over a large range, as shown in Figures 3-7 of paper 1. This gradient is largest for the subsolar point on Mercury, small for a satellite of Jupiter and negligible for $i=$ $60^{\circ}$. The values of $w_{T}$ were found by weighting $w_{\lambda}$ by a Planck function with $T=T_{r}$ and integrating over wavelength, as discussed in paper 1 .

According to equation (71) of paper $\mathbf{1}$, the medium may be treated approximately as emitting like a surface at the temperature $T_{s}$. Hence the emitted spectral radiance $I_{\lambda}(\mu)$ was converted to an apparent spectral directional emissivity $\varepsilon_{d \lambda}(\mu)$ by dividing by $U_{\lambda}\left(T_{s}\right) / \pi$. The resulting apparent spectral emissivities are shown in Figure 5. Also indicated in Figures 3-5 is the location of the Christiansen wavelength. Figures 3-5 illustrate several interesting conclusions.

First, the emissivity minimum is not at the true center of the reststrahlen band, $\lambda=\lambda_{0}$, but is displaced well toward shorter wavelengths. The reason can be seen by inspection of Figures 3-5. The emissivity minimum corresponds to the maximum in $w_{\lambda}$, which occurs where the real part of the refractive index $n_{r}$ $\ll 1$, but the imaginary part $n_{i}$ is still relatively large, so that the volume scattered term of $w_{\lambda}$ is negligible. To a rough approximation,

$$
S_{c} \sim \frac{\left(n_{r}-1\right)^{2}+n_{i}^{2}}{\left(n_{r}+1\right)^{2}+n_{i}^{2}}
$$

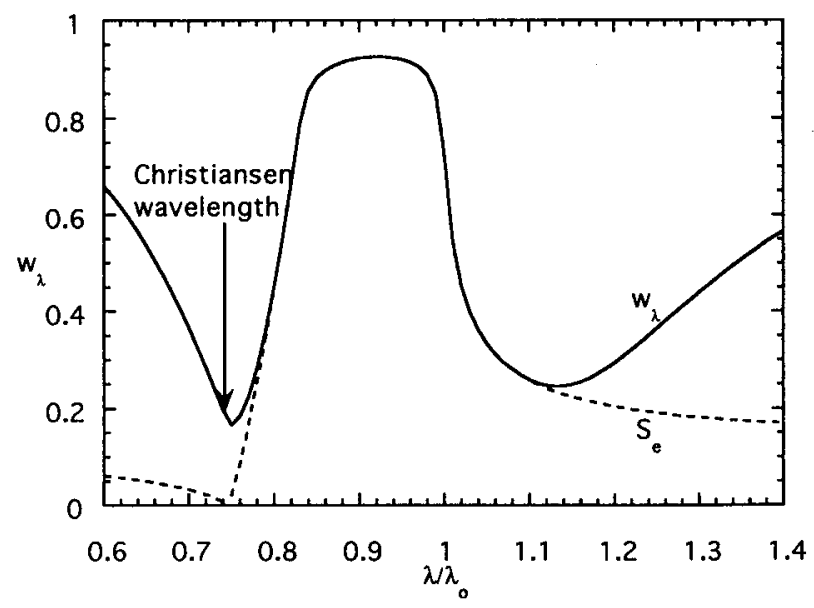

Figure 4. Single scattering albedo spectrum of a spherical particle with $d=10 \lambda_{0}$ and the refractive index shown in Figure $3 b$. Solid line is single scattering albedo; dashed line is contribution of the surface-reflected component, $S_{e}$. 
hence, if $n_{r} \ll 1, S_{e}$ is close to $1, w_{\lambda}$ has a maximum, and $\varepsilon_{d \lambda}(\mu)$ has a minimum.

Second, the short-wavelength 'maximum in apparent emissivity is close to, but not exactly at, the Christiansen wavelength $\lambda_{C}$, which, by definition, occurs where $n_{r}=1.00$. This is clearly seen in Figure 6 , in which the continuum refractive index $n_{c}$ is varied in order to change $\lambda_{C}$. When $n_{c}$ is large, the emissivity maximum is very close to $\lambda_{C}$. However, as $n_{c}$ decreases, $\lambda_{C}$ is increasingly displaced toward shorter wavelengths away from the emissivity maximum.

The traditional argument [Conel, 1969; Salisbury, 1993] is that the medium is transparent there because particle surface scattering is negligible; hence radiation from deeper, hotter layers of the medium reaches the surface, increasing the apparent emissivity. However, Figures 4 and 5 show that the maximum in the emissivity actually occurs at the place that Hapke [1993] has called the first transition minimum in $w_{\lambda}$ and in reflectance. As $\lambda$ increases near this minimum, $n_{i}$ also increases rapidly, until the particle is nearly opaque and the single scattering albedo changes from being dominated by volume-scattered light to being dominated by strong surfacescattered light. Even though the surface scattering is small, the medium is not transparent because the absorption coefficient is not negligible there: at $\lambda=\lambda_{C}, \alpha d=1.26$.

Fortuitously, this first transition minimum always occurs close to $\lambda_{C}$. Hence it is appropriate to continue to refer to the associated emissivity maximum as the "Christiansen maximum," especially since this terminology is now ingrained in the literature. However, this maximum would occur even if $n_{r}$ did not go through 1.00 anywhere and even in the absence of any subsurface temperature gradients.

Third, there is another maximum on the long-wavelength side of the emissivity minimum. Salisbury and Walter [1989] have called this the "transparency feature" and attributed it to a second region of relative transparency of the medium between two reststrahlen bands. However, Figures 4 and 5 show that this maximum is actually caused by a second transition minimum in $w_{\lambda}$, where the particle albedo changes from being dominated by strong surface scattering to being dominated by volume scattering as $n_{i}$ decreases with increasing wavelength

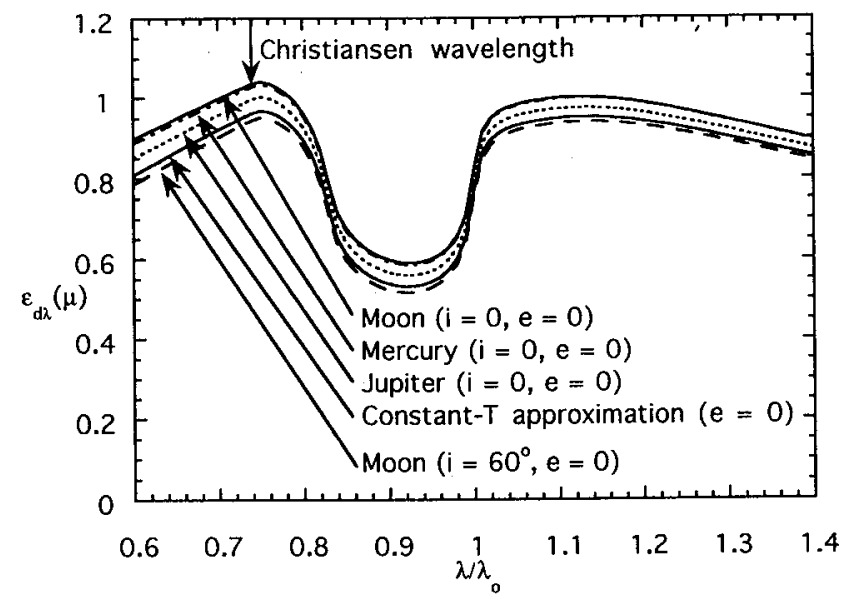

Figure 5. Apparent emissivity spectra of a regolith composed of isotrotropically scattering particles with the spectral single scattering albedo shown in Figure 4 , in equilibrium with sunlight, and illuminated and observed under different conditions.

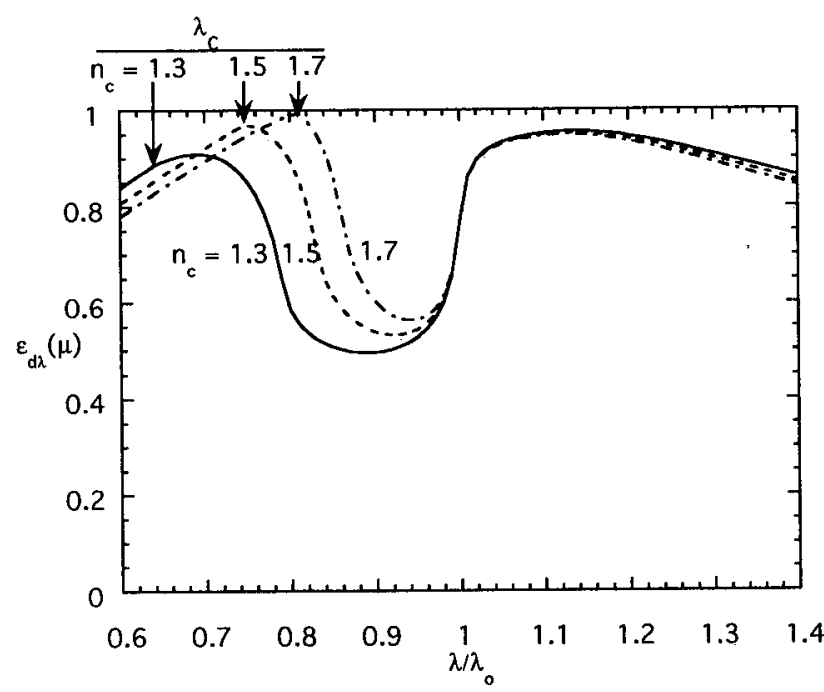

Figure 6. Emissivity spectra, showing the effect of varying the continuum real refractive index $n_{c}$. The arrows indicate the wavelengths of the corresponding Christiansen wavelengths $\lambda_{C}$. Note the displacement of $\lambda_{C}$ from the emissivity maximum.

[Salisbury, 1993; Hapke, 1993]. It occurs even in the absence of a second absorption band. The wavelength at which the transparency maximum occurs is much less sensitive to $n_{c}$ than the Christiansen maximum.

Salisbury and Walter [1989] have noted a strong correlation between the wavelengths of the emissivity minimum and the two maxima. Such a correlation is to be expected because all three are associated with the reststrahlen band. Thus all should be reliable indicators of composition, especially if they can be resolved in the same spectrum. The widths of the two maxima should depend on particle size: as the particle size increases, both the Christiansen maximum and the transparency feature should increase in width.

Fourth, a large subsurface temperature gradient causes the Christiansen maximum to shift toward shorter wavelengths because of the increasing radiance at short wavelengths from the Planck function. However, the amount of the shift is small. This confirms the previous conclusion of B. Henderson and B. Jakosky (submitted manuscript, 1995), and is also consistent with laboratory measurements by Salisbury et al. [1994b]. The reason for the lack of sensitivity is that the particles are not particularly transparent there, as previously thought.

Fifth, the main effect of the subsurface gradient is to raise or lower the emissivity nearly uniformly over the entire wavelength region. This is why the apparent emissivity exceeds unity for some of the curves. However, in practice, this should have little effect. Because of calibration difficulties, absolute emissivity is seldom measured. Usually, emissivity is arbitrarily normalized to unity at the Christiansen maximum.

Also shown in Figure 5 is the effective spectral directional emissivity,

$$
\varepsilon_{\lambda d}(\mu) \simeq \frac{\zeta_{\lambda} \gamma_{\lambda}}{1+2 \zeta_{\lambda} \gamma_{\lambda} \mu} \frac{\left[1+2 \zeta_{\lambda}^{2} \mu\right]+\beta_{\lambda}\left[1-2 \gamma_{\lambda} / \zeta_{\lambda}-2 \gamma_{\lambda}^{2} \mu\right]}{1-\beta_{\lambda}}
$$

which can readily be derived by obvious wavelength-dependent extension of equation (71b) of paper 1. Equation (71b) of 
paper 1 was derived using the boundary layer approximation, and also assuming a constant subsurface temperature. In Figure 5 it was assumed that $\beta_{\lambda}=0$, in which case, equation (26) is

$$
\varepsilon_{\lambda d}(\mu) \simeq \gamma_{\lambda} \frac{1+2 \mu}{1+2 \gamma_{\lambda} \mu} .
$$

Note that its shape is virtually identical to those of the other curves in Figure 5. Hence, except in the unusual circumstance that absolute emissivity is required, the approximate expressions (26) or (27) may be reliably used to infer or calculate the IR parameters of the medium.

\section{Conclusions}

Using a new model [Hapke, this issue] that simultaneously solves the equation of radiative transfer for visible and thermal radiation and the heat equation, three problems of interest in the thermal properties of planetary regoliths were studied.

The solid-state greenhouse effect is shown to be self-limiting because of the strong correlation between optical absorption and albedo and because radiative thermal conductivity is important in a porous regolith, even at low temperatures. If the optical properties of terrestrial snow are representative of those of the regoliths of satellites in the outer solar system, the maximum rise in temperature that may be expected due to a greenhouse effect is about $40 \mathrm{~K}$.

The contribution to thermal beaming caused by nonLambertian albedos and emissivities of a macroscopically smooth regolith was estimated to be able to account for a thermal beaming parameter of about 0.95 , whereas the observed value is about 0.75 . Since coherent backscatter or shadow hiding opposition effects cannot occur in thermal radiation from smooth regoliths of small particles, it is likely that the remainder of the beaming factor is caused by macroscopic roughness.

The nature of features in the emissivity spectrum of a reststrahlen band was investigated using an artificial absorption band generated by the Lorentz equation. It was found that the peak that occurs on the short wavelength side of a spectral emissivity band has been incorrectly identified as denoting the Christiansen wavelength. This was a natural error, because the wavelength of the maximum is close to, but not exactly at, the Christiansen wavelength. The emissivity peak corresponds to one of two transition minima in reflectance, where the radiation scattered by a soil particle changes from volume scattering to surface scattering. The other transition minimum occurs on the long wavelength side of the band and corresponds to the transparency feature seen in emissivity. Because the particles of the regolith are not transparent at either peak, subsurface temperature gradients have only a minor effect on the wavelengths of their locations. Hence the wavelengths of the two peaks and the included minimum should be reliable diagnostic indicators of composition.

Acknowledgments. This work benefited from constructive suggestions by Paul Helfenstein and an anonymous reviewer. It is sponsored by a grant from the Planetary Geology and Geophysics Program, Solar System Exploration Division, National Aeronautics and Space Administration.

\section{References}

Born, M., and E. Wolf, Principles of Optics, 6th ed., Pergamon, Tarrytown, N.Y., 1980.

Brown, R. H., and D. Matson, Thermal effects of insolation propagation into the regoliths of airless bodies, Icarus, 72, 84-94, 1987.

Conel, J., Infrared emissivities of silicates: Experimental results and a cloudy atmosphere model of spectral emission from condensed particulate mediums, J. Geophys. Res., 74, 1614-1634, 1969.

Fanale, F., J. Salvail, D. Matson, and R. H. Brown, The effect of volume phase changes, mass transport, sunlight penetration and densitification on the thermal regime of icy regoliths, Icarus, 88 , 193-204, 1990.

Hapke, B., Theory of Reflectance and Emittance Spectroscopy, Cambridge Univ. Press, New York, 1993.

Hapke, B., A model of radiative and conductive energy transfer in planetary regoliths, J. Geophys. Res., this issue.

Henderson, B., and B. Jakosky, Near-surface thermal gradients and their effects on mid-infrared emission spectra of planetary surfaces, J. Geophys. Res., 99, 19,063-19,074, 1994.

Irvine, W., and J. Pollack, Infrared optical properties of water and ice spheres, Icarus, 8, 324-360, 1968.

Lane, A., and W. Irvine, Monochromatic phase curves and albedos for the lunar disk, Astron. J., 78, 267-277, 1973.

Lebofsky, L., M. Sykes, E. Tedesco, G. Veeder, D. Matson, R. H. Brown, J. Gradie, M. Feierberg, and R. Rudy, A refined "standard" thermal model for asteroids based on observations of 1 Ceres and 2 Pallas, Icarus, 68, 239-251, 1986.

Logan, L., and G. Hunt, Emission spectra of particulate silicates under simulated lunar conditions, J. Geophys. Res., 75, 6539-6548, 1970.

Matson, D., and R. H. Brown, Solid state greenhouses and their implications for icy satellites, Icarus, 77, 67-81, 1989.

McGuire, A., and B. Hapke, An experimental study of light scattering by large, irregular particles, Icarus, 113, 134-155, 1995.

Morrison, D., and L. Lebofsky, Radiometry of asteroids, in Asteroids, edited by T. Gehrels, pp. 184-205, Univ. of Ariz. Press, Tucson, 1979.

Saari, J., and R. Shorthill, The sunlit lunar surface, I, Albedo studies and full moon temperature distribution, Moon, 5, 161-178, 1972.

Saari, J., R. Shorthill, and D. Winter, The sunlit lunar surface, II, A study of far infrared brightness temperatures, Moon, 5, 179-199, 1972.

Salisbury, J., Mid-infrared spectroscopy: Laboratory data, in Remote Compositional Analysis, edited by C. Pieters and P. Englert, pp. 79-98, Cambridge Univ. Press, New York, 1993.

Salisbury, J., and L. Walter, Thermal infrared $(2.5-13.5 \mu \mathrm{m})$ spectroscopic remote sensing of igneous rock types on particulate planetary surfaces, J. Geophys. Res., 94, 9192-9202, 1989.

Salisbury, J., D. D'Aria, and A. Wald, Measurements of thermal infrared spectral reflectance of frost, snow, and ice, J. Geophys. Res., $99,24,235-24,240,1994 \mathrm{a}$.

Salisbury, J., A. Wald, and D. D'Aria, Thermal-infrared remote sensing and Kirchhoff's law, 1, Laboratory measurements, J. Geophys. Res., 99, 11,897-11,911, 1994b.

Schwerdtfeger, P., and G. Weller, Radiative heat transfer processes in snow and ice, Meteorol. Stud. Plateau Stn., Antarct., 25, 35-39, 1977.

Sinton, W., Temperatures on the lunar surface, in Physics and Astronomy of the Moon, edited by Z. Kopal, pp. 407-428, Academic, San Diego, Calif., 1961.

Spencer, J., A rough-surface thermosphysical model for airless planets, Icarus, 83, 27-38, 1990.

Spitzer, W., and D. Kleinman, Infrared lattice bands of quartz, Phys. Rev., 121, 1324-1335, 1961.

Wald, A., and J. Salisbury, Thermal infrared directional emissivity of powdered quartz, J. Geophys. Res., 100, 24,665-24,675, 1995.

Wooten, F., Optical Properties of Solids, Academic, San Diego, Calif., 1972.

B. Hapke, 321 Old Engineering Hall, University of Pittsburgh, Pittsburgh, PA 15260. (e-mail: hapke@vms.cis.pitt.edu)

(Received May 9, 1995; revised March 18, 1996; accepted March 20, 1996.) 